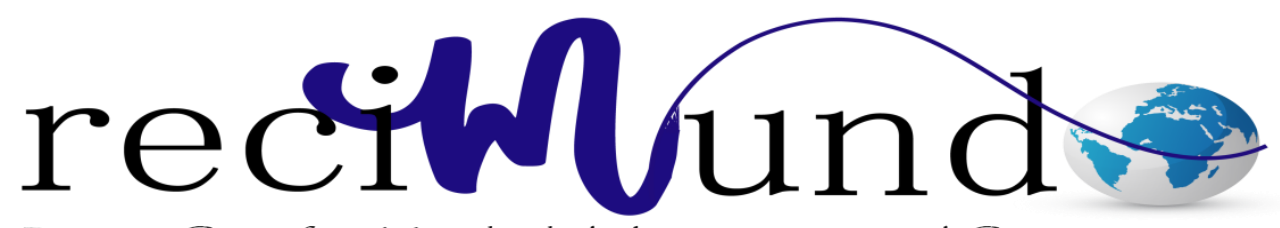

Revista Científica Mundo de la Investigación y el Conocimiento

Tifany Zamora Zambrano ${ }^{\text {a; }}$ Jessica Pesantes Salazar ${ }^{\text {b; }}$

Ivonne Yaguarema Pincay ${ }^{\text {c; }}$ Silvia Villagómez Vera ${ }^{\mathrm{d}}$

Aplicación del procedimiento al ingreso del paciente por el profesional de enfermería área de emergencia de un hospital pediátrico

Revista Científica Mundo de la Investigación y el Conocimiento. Vol. 2 núm., 1, febrero, ISSN: 2588-073X, 2018, pp. 146-156

DOI: $10.26820 /$ recimundo/2.1.2018.146-156

Editorial Saberes del Conocimiento

Recibido: 05/12/2017

Aceptado: 10/02/2018
a. Junta de Beneficencia de Guayaquil; tzamora@jbgye.org.ec
b. Junta de Beneficencia de Guayaquil; jpesantess@jbgye.org.ec
c. Junta de Beneficencia de Guayaquil; iyaguarema@jbgye.org.ec
d. Junta de Beneficencia de Guayaquil; svillagomez@jbgye.org.ec 


\section{Aplicación del procedimiento al ingreso del paciente por el profesional de enfermería área de emergencia de un hospital pediátrico}

Vol. 2, núm. 1., (2018)

Tifany Zamora Zambrano; Jessica Pesantes Salazar; Ivonne Yaguarema Pincay; Silvia

Villagómez Vera

\section{RESUMEN}

El profesional de enfermería cumple diferentes funciones donde una de las primordiales es su participación al ingreso del paciente lo que ayudara a mantener una integración ,enfermera, paciente y familia para recuperar su bienestar biopsicosocial. El presente trabajo se lo considera de investigación acción porque tiene tres etapas: Diagnostica, Intervención y Evaluación. La Diagnostica tiene como objetivo Identificar la aplicación del procedimiento al ingreso del paciente por el profesional de enfermería en el área de emergencia. Se fundamenta en el Modelo de Virginia Henderson que refiere cuidados básicos de enfermería apoyándose en la fisiología y psicología, la Teoría del Cuidado Humano de Jean Watson quien estudia las humanidades expande la mente e incrementa la capacidad de pensar y el desarrollo personal y el Modelo de los Procesos Vitales de Martha Rogers el objetivo es procurar y promover una interacción armónica entre el hombre y su entorno. El enfoque metodológico es cualicuantitativo, inductivo-deductivo, descriptivo, transversal. El instrumento y técnica de obtención de datos es la encuesta, observación participativa y grupo focal. Los resultados que se obtienen según la encuesta es que el 100\% se lavan las mano, verifica la identificación del paciente, se presenta al paciente, interacción enfermera y/o paciente - familiar, informa y facilita al paciente y/o familiar sobre los derechos y deberes de los mismos, estos datos son altos , pero no tienen relación con los resultados de la observación participativa y grupo focal. En base a estos resultados, se realiza la intervención y evaluación, para lo cual, se reestructura el protocolo, se capacita al personal de enfermería y realizan evaluaciones continuas.

Palabras clave: Enfermería, procedimiento, protocolo, modelo. 


\section{Aplicación del procedimiento al ingreso del paciente por el profesional de enfermería área de emergencia de un hospital pediátrico}

Vol. 2, núm. 1., (2018)

Tifany Zamora Zambrano; Jessica Pesantes Salazar; Ivonne Yaguarema Pincay; Silvia Villagómez Vera

\section{ABSTRACT}

The nurse performs different functions where one of the most important is their participation at the patient's entrance, which will help maintain an integration, nurse, patient and family to recover their biopsychosocial well-being. The present work is considered as an action research because it has three stages: Diagnosis, Intervention and Evaluation. The objective of the Diagnostic is to identify the application of the procedure upon patient admission by the nursing professional in the emergency area. It is based on the Virginia Henderson Model that refers basic nursing care based on physiology and psychology, the Theory of Human Care by Jean Watson, who studies the humanities, expands the mind and increases the capacity for thinking and personal development and the Model of Vital Processes of Martha Rogers the objective is to seek and promote a harmonious interaction between man and his environment. The methodological approach is quali-quantitative, inductive-deductive, descriptive, and transversal. The instrument and technique of obtaining data is the survey, participatory observation and focus group. The results obtained according to the survey is that $100 \%$ wash their hands, verify the identification of the patient, present the patient, nurse interaction and / or patient - family, inform and facilitate the patient and / or family about the rights and their duties, these data are high, but they are not related to the results of the participatory observation and focal group. Based on these results, the intervention and evaluation is carried out, for which the protocol is restructured, the nursing staff is trained and continuous evaluations are carried out.

Keywords: Nursing, procedure, protocol, model. 


\section{Aplicación del procedimiento al ingreso del paciente por el profesional de enfermería área de emergencia de un hospital pediátrico}

Vol. 2, núm. 1., (2018)

Tifany Zamora Zambrano; Jessica Pesantes Salazar; Ivonne Yaguarema Pincay; Silvia

Villagómez Vera

\section{Introducción.}

El profesional de enfermería cumple diferentes funciones donde una de las primordiales es su participación al ingreso del paciente lo que ayudara a mantener una integración ,enfermera, paciente y familia para recuperar su bienestar biopsicosocial. $(1,2)$

A nivel mundial y a nivel de país no existen datos estadísticos pero se puede verificar de otros estudios realizados sobre la calidad de cuidados en hospitales de Latinoamérica, donde expresan los cuidados de enfermería brindados a pacientes hospitalizados en los servicios de medicina interna, hospitalización y urgencias, la profesional de enfermería se encuentra en un nivel medio respecto a la suficiencia y un nivel regular con respecto al índice de precisión. $(3,4,5)$

Esta investigación tiene como objetivo Identificar la aplicación del procedimiento al ingreso del paciente por el profesional de enfermería en el área de emergencia de un hospital pediátrico de la ciudad de Guayaquil, cabe indicar que los profesionales conocen los procedimientos pero no lo aplican adecuadamente y esto puede depender de factores como: el desconocimiento de la existencia del manual en el área, falta de tiempo, demanda de pacientes, déficit de recursos materiales, stress laboral.

\section{Metodología.}

El diseño de la investigación a realizar en este estudio es cuali-cuantitativa. Se considera cuantitativa porque incluye una medición sistemática de los Procedimientos de Enfermería al ingreso de paciente y se emplea un análisis estadístico del mismo acorde al tiempo de los hechos 


\section{Aplicación del procedimiento al ingreso del paciente por el profesional de enfermería área de emergencia de un hospital pediátrico}

Vol. 2, núm. 1., (2018)

Tifany Zamora Zambrano; Jessica Pesantes Salazar; Ivonne Yaguarema Pincay; Silvia Villagómez Vera

empleando la modalidad deductiva la misma que se verá reflejada en gráficos posterior a la aplicación de la encuesta. Es cualitativa porque permite describir costumbres, pensamientos y percepciones del profesional de enfermería y pacientes para darles un significado. Se realiza una Observación Participativa a los profesionales con la finalidad de conocer las aptitudes ante el paciente durante su ingreso mientras que a los padres de familia se les realiza un Grupo Focal para saber que piensan ellos sobre el trabajo del profesional de enfermería, además de ser, de modalidad inductiva porque permite medir las cualidades.

Se considera una investigación de tipo Prospectivo porque se registra la información según se suscitan, según el periodo y la secuencia del trabajo, transversal porque el tiempo no es importante en relación con la forma como se suscitan los hechos, descriptiva porque permitió detallar los sucesos como son observados y comparativas por que se tiene una línea base.

\section{Población y muestra}

La población: 290 de profesionales de enfermería

Muestra: Sera no pirobalística por conveniencia, porque sean considerado 22 profesionales de enfermería del área de emergencia.

\section{Instrumento}

El instrumento ejecutado en esta investigación cuantitativa es la encuesta, la misma que nos permite obtener y elaborar datos con la finalidad de valorar el conocimiento de los profesionales sobre el cuidado del paciente a su ingreso. 


\section{Aplicación del procedimiento al ingreso del paciente por el profesional de enfermería área de emergencia de un hospital pediátrico}

Vol. 2, núm. 1., (2018)

Tifany Zamora Zambrano; Jessica Pesantes Salazar; Ivonne Yaguarema Pincay; Silvia

Villagómez Vera

Y la técnica utilizada en la investigación cualitativa es la Observación Participativa que ayuda a conocer y recopilar información directamente del profesional de enfermería que está en contacto con el paciente a su ingreso. Se realiza grupo focal dirigido a profesionales y familiares

\section{Prueba piloto}

Se aplica a 6 profesionales en áreas hospitalarias, a los mismos que se realizó la encuesta con la finalidad de obtener su especificad y validez poder aplicar la misma en el área descrita siendo, como resultado se obtuvo que las preguntas son claras y entendibles para responder.

Recolección de la información

Se realiza en un mes aplicando las siguientes dimensiones como : conocimiento cuyo objetivo es de valorar al profesional de enfermería; materiales, equipos a verificar aseo y disponibilidad del mismo y factores que influyen en el cuidado al ingreso del paciente .

Este instrumento se aplica a 22 profesionales utilizando la técnica de observación en base a un cronograma se realizara en 3 días. 
Aplicación del procedimiento al ingreso del paciente por el profesional de enfermería área de emergencia de un hospital pediátrico

Vol. 2, núm. 1., (2018)

Tifany Zamora Zambrano; Jessica Pesantes Salazar; Ivonne Yaguarema Pincay; Silvia Villagómez Vera

\section{Resultados.}

Datos comparativos de los resultados de la investigación cuantitativa y cualitativa para realizar el análisis (Método de Triangulación)

\begin{tabular}{|c|c|c|c|c|c|c|c|c|}
\hline DATOS & \multicolumn{5}{|c|}{$\begin{array}{l}\text { OBSERVACIÓN PARTICIPATIVA } \\
\text { (CUALITATIVA) }\end{array}$} & \multicolumn{2}{|c|}{$\begin{array}{l}\text { GRUPO FOCAL } \\
\text { (CUALITATIVA) }\end{array}$} & $\begin{array}{c}\text { ENCUESTA } \\
\text { (CUANTITATI } \\
\text { VA) }\end{array}$ \\
\hline \multirow[b]{2}{*}{ ACTIVIDADES } & \multicolumn{4}{|c|}{ ESCALAS } & \multirow[b]{2}{*}{ TOTAL } & \multirow[b]{2}{*}{ Expresiones } & \multirow[b]{2}{*}{$\begin{array}{l}\text { Núcleo del } \\
\text { sentido }\end{array}$} & \multirow[b]{2}{*}{$\%$} \\
\hline & $\begin{array}{l}\text { Lo } \\
\text { hace }\end{array}$ & $\begin{array}{l}\text { No lo } \\
\text { hace }\end{array}$ & $\begin{array}{c}\text { A } \\
\text { vece } \\
\mathbf{S}\end{array}$ & $\begin{array}{c}\text { Nun } \\
\text { ca }\end{array}$ & & & & \\
\hline $\begin{array}{l}\text { Se presenta al paciente } \\
\text { y familia }\end{array}$ & & 3 & 9 & 10 & 22 & $\begin{array}{l}\text { Yo saludo y } \\
\text { pregunto qué le } \\
\text { paso }\end{array}$ & $\begin{array}{l}\text { Interacción/ } \\
\text { comunicación } \\
\text { con el } \\
\text { paciente/ } \\
\text { familiar. }\end{array}$ & 100 \\
\hline $\begin{array}{l}\text { Comunicación entre } \\
\text { enfermera y/o paciente - } \\
\text { familiar (Empatía) }\end{array}$ & 2 & & 20 & & 22 & $\begin{array}{l}\text { Yo me presento } \\
\text { y si el niño está } \\
\text { estable me } \\
\text { pongo a } \\
\text { conversar con él } \\
\text { le pregunto si } \\
\text { está en la } \\
\text { escuela, lo } \\
\text { entretengo y le } \\
\text { explico lo que le } \\
\text { voy hacer, pero } \\
\text { si es un niño que } \\
\text { viene } \\
\text { convulsionando } \\
\text { bueno canalizo } \\
\text { la vía y después } \\
\text { se conversa con } \\
\text { la mamá. }\end{array}$ & $\begin{array}{l}\text { Interacción } \\
\text { con el } \\
\text { paciente/ } \\
\text { Familiar. }\end{array}$ & 100 \\
\hline Lavado de manos & 10 & & 12 & & 22 & $\begin{array}{l}\text { Ninguna } \\
\text { Respuesta }\end{array}$ & Sin respuesta & 100 \\
\hline $\begin{array}{c}\text { Verifica y coloca la } \\
\text { identificación al } \\
\text { paciente }\end{array}$ & 7 & 2 & 5 & 8 & 22 & $\begin{array}{c}\text { Este.., } \\
\text { identificación } \\
\text { del paciente } \\
\text { llamarlos por }\end{array}$ & $\begin{array}{l}\text { Identificación } \\
\text { del paciente }\end{array}$ & 100 \\
\hline
\end{tabular}




\section{Aplicación del procedimiento al ingreso del paciente por el profesional de enfermería área de emergencia de un hospital pediátrico}

Vol. 2, núm. 1., (2018)

Tifany Zamora Zambrano; Jessica Pesantes Salazar; Ivonne Yaguarema Pincay; Silvia

Villagómez Vera

\begin{tabular}{|c|c|c|c|c|c|c|c|c|}
\hline & & & & & & $\begin{array}{c}\text { apellidos, en si } \\
\text { nosotros no } \\
\text { colocamos los } \\
\text { brazales lo } \\
\text { coloca el } \\
\text { admisionista o } \\
\text { se lo dan a la } \\
\text { chica. auxiliares }\end{array}$ & & \\
\hline $\begin{array}{l}\text { Informa y facilita al } \\
\text { paciente y/o familiar } \\
\text { sobre los derechos y } \\
\text { deberes de los mismos }\end{array}$ & & 2 & 15 & 5 & 22 & $\begin{array}{l}\text { Las indicaciones } \\
\text { al ingreso que } \\
\text { tiene que estar } \\
\text { un solo familiar } \\
\text { de lo que le voy } \\
\text { hacer al niño, } \\
\text { eso si se hace y } \\
\text { todas lo } \\
\text { hacemos. }\end{array}$ & $\begin{array}{l}\text { Información } \\
\text { breve de las } \\
\text { reglas del área }\end{array}$ & 86 \\
\hline $\begin{array}{l}\text { Muestra el área física al } \\
\text { paciente y enseña su } \\
\text { correcta utilización }\end{array}$ & & 1 & 14 & 7 & 22 & $\begin{array}{l}\text { Ninguna } \\
\text { respuesta }\end{array}$ & Sin respuesta & 100 \\
\hline $\begin{array}{c}\text { Dispone de } \\
\text { material(camas o cunas ) } \\
\text { limpias al ingreso del } \\
\text { paciente }\end{array}$ & & 1 & 15 & 6 & 22 & $\begin{array}{c}\text { Son los } \\
\text { monitores, } \\
\text { sabanas, camas, } \\
\text { proporcionar } \\
\text { todo lo que va } \\
\text { utilizar el } \\
\text { paciente. }\end{array}$ & $\begin{array}{l}\text { Disponibilida } \\
\mathrm{d}\end{array}$ & 82 \\
\hline $\begin{array}{c}\text { Acompañar y acomodar } \\
\text { al paciente y familia en la } \\
\text { unidad }\end{array}$ & & 5 & 10 & 7 & 22 & $\begin{array}{c}\text { Si se acompaña } \\
\text { al familiar según } \\
\text { la demanda de } \\
\text { pacientes. }\end{array}$ & Lo hace & 100 \\
\hline $\begin{array}{l}\text { Registrar en la historia } \\
\text { clínica electrónica del } \\
\text { paciente }\end{array}$ & 22 & & & & 22 & $\begin{array}{l}\text { Sí, Todo el } \\
\text { trabajo se ve } \\
\text { reflejado en el } \\
\text { sistema }\end{array}$ & Se realiza & 100 \\
\hline
\end{tabular}




\section{Aplicación del procedimiento al ingreso del paciente por el profesional de enfermería área de emergencia de un hospital pediátrico}

Vol. 2, núm. 1., (2018)

Tifany Zamora Zambrano; Jessica Pesantes Salazar; Ivonne Yaguarema Pincay; Silvia Villagómez Vera

\section{Conclusiones}

Una vez que se tomaron los datos y se realizó el análisis e interpretación de los mismos se llegó a las siguientes conclusiones:

Los profesionales de enfermería en estudio conoce los procedimiento al ingreso del paciente pero no se aplica adecuadamente lo siguiente: presentación al paciente/familiar, lavado de manos, verificación de la identificación del paciente, reconocimiento del área física.

En cuanto al material y equipo que debe proveer el profesional de enfermería en muchas ocasiones es deficiente por la demanda de pacientes tales como: camas, cunas, sabanas, bombas de infusión;

Son varios los factores que influye en el profesional para brindar adecuados cuidados de enfermería al ingreso pero se a considerado que el estrés laboral (síndrome de Burnout), es el que más afecta para poder brindar este cuidado.

La percepción de los padres de familia al ingreso refieren la empatía existente entre enfermera - paciente/familia.

La aplicación del procedimiento al ingreso del paciente por el profesional de enfermería en el área de emergencia se cumple parcialmente. Siendo evidente en el método de triangulación habiendo una disociación de resultados en el estudio cuantitativo y cualitativo indica un $100 \%$ en la realización de los procedimiento por parte del profesional : presentación al paciente/familiar, lavado de manos, verificación de la identificación del paciente, 


\section{Aplicación del procedimiento al ingreso del paciente por el profesional de enfermería área de emergencia de un hospital pediátrico}

Vol. 2, núm. 1., (2018)

Tifany Zamora Zambrano; Jessica Pesantes Salazar; Ivonne Yaguarema Pincay; Silvia Villagómez Vera

reconocimiento del área física. Mientras que en la parte cualitativa los resultados son diferentes.

\section{Recomendaciones.}

En base a las conclusiones obtenidas se realizan las siguientes recomendaciones:

- La reestructuración del Protocolo al Ingreso del paciente en el área de Emergencia.

- Educación permanente a los profesionales de enfermería sobre el protocolo.

- Evaluación permanente a los profesionales de enfermería sobre el protocolo.

- Actualizar el protocolo de acuerdo a las necesidades.

- Crear un comité para la actualización del manual de procedimiento existente en el área.

\section{Bibliografía.}

1. Wesley R. Teorías y modelos de enfermería México: Mcgraw-Hill Interamericana; 1997.

2. Hernández Conesa J. Historia de la enfermería. un análisis histórico de los cuidados de enfermería Madrid: Mcgraw-hill Interamericana; 1995.

3. Santana de Freitas J, Bauer de Camargo Silva AE, Minamisava R, Queiroz Bezerra AL, Gomes de Sousa MR. Calidad de los cuidados de enfermería y satisfacción del paciente atendido en un hospital de enseñanza. Rev. Latino-Am. Enfermagem. 2014 Jun; 22(3): p. 454460 .

4. Hernández Terrazas LE, Díaz Oviedo A, Martinez Licona JF, Hernandez DG. Educación de 


\section{Aplicación del procedimiento al ingreso del paciente por el profesional de enfermería área de emergencia de un hospital pediátrico}

Vol. 2, núm. 1., (2018)

Tifany Zamora Zambrano; Jessica Pesantes Salazar; Ivonne Yaguarema Pincay; Silvia Villagómez Vera

enfermería en el cuidado humanizado. Esc Anna Nery. 2018 Ene; 22(1): p. e20170275.

5. Raile Alligood M. Modelos y Teorias En Enfermeria. 7th ed. Madrid: Elsevier; 2011. 